\title{
LE TRANSIT EPIDIDYMO-DEFERENTIEL
}

\author{
Jean-Pierre DADOUNE
}

\author{
Service de Biologie de la Fertilité et de Cytogénétique - CECOS \\ Hôtel-Dieu de Paris \\ 1, place du Parvis Notre-Dame - 75181 PARIS CEDEX 04
}

\section{SPERM TRANSPORT THROUGH THE EPIDIDYMIS AND VAS DEFERENS.}

The epididymis and vas deferens constitute not only a simple conduit for sperm transport but also play an important physiological role in the development of sperm fertilizing ability. The epithelial compartment plays a major functional role in determining the biochemical composition of the luminal fluid in which the spermatozoa undergo a series of structural, biochemical and metabolic changes. During epididymal transit spermatozoa acquire their capacity for motility and also their ability to attach and bind to the zona pellucida and fertilize the oocyte. In man, sperm maturation may occur in the extreme proximal region of the epididymis. The regulation of epididymal and vasa deferential function, as well as sperm maturation, are under androgenic control. Key words : epididymis - Vas deferens - Physiology - Sperm maturation Andrologie, 1992, $2:$ :44-47

Le conduit épididymo-déférentiel assure non seulement le transport mais aussi l'acquisition du pouvoir fécondant des spermatozoïdes. En dépit des interrogations suscitées sur le rôle effectif de l'épididyme par l'obtention de grossesses avec des spermatozoïdes en provenance de la tête épididymaire (13,21), il est clairement établi que le transit des spermatozoïdes dans l'épididyme intact accroit notablement le pourcentage des formes mobiles ainsi que leur aptitude à se fixer sur la zone pellucide (6).

Les modifications biochimiques, métaboliques et fonctionnelles qui rendront les spermatozoïdes aptes à la fécondation sont directement en relation avec les fonctions métaboliques de l'épithélium épididymaire et la composition du fluide épididymaire. Les facteurs élaborés par l'épididyme et le développement du pouvoir fécondant des spermatozoides sont sous la dépendance des androgènes $(2,5,16,17)$.

Le canal déférent assure le stockage des spermatozoïdes et participe activement à leur expul- sion dans les canaux éjaculateurs. Egalement androgéno-dépendant il pourrait jouer un rôle physiologique complémentaire dans la maturation des spermatozoïdes (4).

\section{TRANSPORT, STOCKAGE ET ÉMISSION DES SPERMATOZOIDES}

Le flux des spermatozoïdes pénétrant dans l'épididyme est constant chez les mammifères. Il s'effectue sous le contrôle de la pression positive du liquide du rete testis, des courants liquidiens déterminés par les battements des cils des cellules épithéliales de la paroi des canaux efférents et les contractions péristaltiques du manchon de cellules musculaires lisses.

Chez l'homme, après incorporation de thymidine-H3 à des volontaires, la durée du transit épididymaire a été estimée à 1 jour environ dans la tête et à un peu plus de 5 jours dans l'ensemble corps-queue, l'apparition des spermatozoïdes marqués dans l'éjaculat pouvant s'échelonner entre 1 et 12 jours (19). Cependant, quand la durée a été évaluée d'après les réserves spermatiques dans les différentes portions épididymaires, le temps de transport est apparu nettement raccourci par rapport aux autres espèces: de 1 à 2 jours pour le transport total, et d'un demi-jour pour le trajet tête-corps (1).

La progression des spermatozoïdes dans le canal épididymaire est contrôlée par deux facteurs: la pression hydrostatique intraluminale et les contractions des cellules musculaires lisses de la tunique musculaire. En complément des mécanismes cholinergiques, adrénergiques et de l'action de la vasopressine, les prostaglandines interviennent dans la régulation de l'activité contractile de la tunique musculaire : la fréquence et l'amplitude des contractions sont augmentées par PGF2 $\alpha$ et, au contraire, diminuées par PGE2.

Cinquante pour cent environ des spermatozoïdes restent vivants en atteignant la queue où ils sont stockés, parfois plus de 3 semaines. Chez l'homme, la réserve en spermatozoildes dans cette région est à peu près égale au nombre des spermatozoïdes éjaculés, qu'il soit ou non en repos sexuel (1). Il faut noter que la mobilité des sper- matozoïdes se trouve réduite du fait de la température qui est nettement inférieure à la température centrale. Un séjour prolongé dans la queue entraîne une baisse du pouvoir fécondant. Les spermatozoïdes non éjaculés peuvent être éliminés dans l'urine, Iysés ou résorbés à l'intérieur de l'épididyme. La fonction de spermiophagie serait assurée soit par les cellules épithéliales de la paroi soit par les macrophages disséminés dans la lumière. Un excès de spermatozoides ou la présence d'un nombre élévé de formes atypiques aurait pour conséquence d'accroître la spermiophagie.

Le transport des spermatozoïdes de la queue de l'épididyme vers le canal déférent est lent et continu. Le canal déférent a la capacité de stocker environ $70 \%$ des spermatozoïdes destinés à une seule éjaculation. La fonction de spermiophagie est également exercée par les cellules épithéliales de l'ampoule déférentielle (14). Au moment de l'éjaculation, les spermatozoïdes sont expulsés dans les canaux éjaculateurs par des contractions puissantes et brèves engendrées par la stimulation adrénergique du manchon musculaire de la queue distale épididymaire et du canal déférent. Des récepteurs de la DHT ont été détectés en abondance dans les cellules musculaires lisses. L'augmentation de la contractilité de la tunique musculaire après traitement par les androgènes serait en relation avec des modifications dans le nombre, la taille et la proportion relative des cellules musculaires lisses qui recoivent des terminaisons afférentes noradrénergiques (3).

\section{MATURATION DES SPERMATOZOIDES}

La nature canalaire du conduit épididymodéférentiel conduit à distinguer deux compartiments : le compartiment épithélial et le compartiment luminal. Les fonctions du compartiment épithélial jouent un rôle déterminant dans la composition du fluide intraluminal dans lequel les spermatozoïdes en transit poursuivent leur maturation.

\section{Fonctions métaboliques du compartiment épithélial}

a) Synthèse et sécrétion: L'analyse électrophorétique des protéines extraites d'homogénats de 
tissu ou du liquide épididymaire, ainsi que les études radioautographiques, ont démontré que chez l'homme, des protéines sont élaborées et sécrétées par les cellules épithéliales, dans les différents segments du canal épididymaire (12, 18). Au moins chez l'animal, l'aptitude à synthé tiser et à libérer des protéines, de surcroît androgéno-dépendantes, est également le fait de l'épithélium du canal déférent (22).

Quelques protéines reconnues pour avoir un rôle spécifique dans la fertilité ont été identifiées dans l'épididyme chez diverses espèces: "Acidic Epididymal Glycoprotein" -A.E.G.-, "Prealbumin Specific Protein" -PES-, "Specific Epididymal Proteins" -EP1-EP6- impliquées dans la liaison à la zone pellucide, "Acrosome Stabilising Factor" -A.S.F.-, "Forward Motility Protein" -FMP-, ou "Immobilin" en relation avec la mobilité (7).

A ce jour, chez l'homme, seules des protéines du revêtement membranaire des spermatozoïdes ont été mises en évidence dans les cellules principales et basales de la partie distale de la tête et du corps de l'épididyme. Une fois libérées, elles se lient aux spermatozoïdes (2).

L'épithélium épididymaire est également capable de libérer dans la lumière des ions et des petites molécules organiques comme la carnitine, l'inositol et la glycérylphosphorylcholine.

b) Fonctions d'absorption. Les cellules épithéliales épididymaires ont la faculté de réabsorber le liquide intraluminal et les molécules injectées dans la lumière (marqueurs non spécifiques). Plus de $90 \%$ du liquide sortant du testicule sont réabsorbés dans les canaux efferents et la tête de l'épididyme. Il s'ensuit à ce niveau une augmentation notable de la concentration des spermatozoïdes. Les cellules épithéliales du canal déférent paraissent aussi capables d'endocyter des molécules exogènes et de les transporter de la lumière vers le tissu conjonctif. En outre, les cellules épithéliales de la tête épididymaire peuvent internaliser diverses protéines spécifiques ( $A B P$, transferrine, $\alpha 2$-macroglobuline) suivant le mécanisme d'endocytose médiée par récepteurs. Il a été établi très récemment que le récepteur de l'ABP, localisé sur la membrane plasmique des cellules épithéliales épididymaires, se lie également à la SBP ("Sex Steroïd Binding Protein") (11).

c) Métabolisme des stéroides: Les cellules épithéliales du conduit épididymodéférentiel possèdent en commun la capacité de capter et de métaboliser la testostérone. La conversion de la testostérone en DHT est assurée par la $5 \alpha$ réductase. En revanche, la biosynthèse de testostérone à partir du cholestérol ou des précurseurs stéroïdiens reste controversée.

\section{Composition du liquide épididymaire}

L'intervention de la barrière sang - épididyme sur le transfert de certaines substances du sang vers la lumière canalaire a été mise en évidence par le dosage de composés organiques (Inositol) carnitine, L-glucose, sérum albumine,...) dans le liquide épididymaire prélevé par microponctions étagées après administration sanguine de ces mêmes composés radioactifs.

Le liquide épididymaire renferme de l'eau, des ions, des protéines, des glycoprotéines, des petites molécules organiques, des spermatozoïdes vivants ou en dégénérescence et un matériel particulaire sous forme de vésicules et de corps denses d'origine inconnue.

La plupart des composés organiques mis en évidence dans le tissu épididymaire ou résultant de son activité métabolique sont retrouvés dans le liquide épididymaire. Outre les protéines ou les glycoprotéines épididymaires impliquées dans les remaniements membranaires ou la régulation de la mobilité des spermatozoïdes, des protéines en provenance du testicule ( $\alpha 2$ macroglobuline, transferrine, ABP, clusterine) ont été identifiées.

Bien que de nombreuses enzymes soient libérées dans le liquide épididymaire, le rôle qui leur est attribué dans la maturation des gamètes reste limité (glycosidase, glycosyltransférase). L' $\alpha 1-4$ glucosidase détectée dans les cellules épithéliales du corps et de la queue de l'épididyme (23) est utilisée comme indicateur de la fonction épididymaire chez l'homme, mais son rôle biologique réel n'est pas établi. D'autres enzymes ont été également détectées dans le liquide épididymaire : phosphatase acide, $\mathrm{N}$-acetyl- $\mathrm{B}$-D-glucosaminidase, arylsulfatase, $B$-glucuronidase et en particulier une forme épididyme-spécifique de l'enzyme de conversion de l'angiotensine I.

Les petites molécules organiques sont surtout représentées par l'inositol, la glycerylphosphorylcholine, la carnitine et les stéroïdes. La concentration en carnitine et en acetylcarnitine s'accroit le long de l'épididyme chez toutes les espèces étudiées, l'homme y compris, alors que les taux de glycerylphosphorylcholine sont spécialement élevés chez l'animal. A l'entrée dans l'épididyme, la testostérone est l'androgène le plus abondant, mais la DHT devient prépondérante dans la tête épididymaire, du fait de l'acti- vité de la $5 \alpha$ réductase épithéliale. Durant le transit épididymaire, la concentration de la testostérone se maintient alors que celle de la DHT décroit de la tête à la queue. D'autres petites molécules organiques sont retrouvées à des concentrations variables suivant le niveau épididymaire: acide sialique, glycerol, prostaglandines, glutathion, acide ascorbique et vitamine D.3.

\section{Maturation épididymaire des spermatozoides}

La maturation des spermatozoïdes est définie par l'ensemble des modifications structurales, biochimiques, métaboliques et fonctionnelles qui les rendront aptes à féconder l'ovocyte et à assurer un développement embryonnaire normal.

Les transformations les plus marquantes concernent l'acrosome, le noyau et la membrane plasmique :

- Modification du système préacrosine-acrosine durant le transit épididymaire (15).

- Condensation de la chromatine déterminée par l'augmentation du nombre de liaisons disulfures (9).

- Changement de la composition des lipides membranaires ayant pour effet d'accroitre la stabilité membranaire (5).

- Remaniements de la surface membranaire, en particulier en relation avec l'adjonction de protéines sécrétoires épididymaires (8).

a) Acquisition de la mobilité fléchante : Chez tous les mammifères étudiés, le pourcentage de spermatozoïdes présentant une mobilité progressive s'accroît à partir de la région distale de la tête pour atteindre une valeur maximale dans le corps de l'épididyme. L'acquisition et le contrôle de la mobilité fléchante dépendent de facteurs endogènes (élévation du taux intracellulaire de l'AMP cyclique, diminution du calcium intracellulaire et de l'activité calmoduline, élévation du $\mathrm{pH}$ intracellulaire ) mais aussi de facteurs environementaux. Ainsi une protéine de mobilité ("Forward Mobility Protein" -FMP-), en provenance de la tête épididymaire, pourrait induire une mobilité plus ou moins linéaire. La carnitine et l'acétylcarnitine dont les taux augmentent dans les spermatozoïdes au cours du transit épididymaire seraient des substrats énergétiques nécessaires au maintien de la mobilité progressive.

Chez l'homme, les résultats obtenus après anastomose épididymodéférentielle conduisent à admettre que l'acquisition de la mobilité fléchante survient dans les régions plus proximales de 
l'épididyme. En effet, quand la distance entre le bord proximal du site de l'anastomose et le bord proximal de la tête est comprise entre 10 et 12 $\mathrm{mm}, 40$ à $80 \%$ de spermatozoïdes mobiles sont retrouvés dans l'éjaculat au delà d'une année après l'intervention (20).

b) Acquisition de l'aptitude à se fixer sur la zone pellucide : Les spermatozoïdes humains prélevés dans l'épididyme proximal se révèlent capables "in vitro" de se lier à la zone pellucide isologue (10). Une protéine spécifique a été détectée par immunocytochimie dans les cellules épithéliales de la tête distale et du corps de l'épididyme et sur l'acrosome des spermatozoïdes épididymaires. Chez les hommes inféconds cette protéine est localisée sur la cape post-acrosomale plutôt que sur l'acrosome. D'autre part, des anticorps dirigés contre ces protéines sécrétoires épididymaires entraînent une réduction notable du taux de fécondation des ovocytes de hamster dépellucidés, sans retentir sur l'agglutination, la mobilité ou la réaction acrosomique spontanée des spermatozoïdes (2).

c) Aptitude à féconder l'ovocyte et à assurer un développement normal : Chez la plupart des mammiferes, les spermatozoïdes acquièrent leur pouvoir fécondant dans la région proximale de la queue épididymaire. Même quand la fécondation est obtenue avec des spermatozoïdes en provenance du corps, la segmentation de l'oeuf est retardée et le développement embryonnaire s'arrête. Dans l'espèce humaine, des naissances ont été rapportées après fécondation avec des spermatozoïdes de la partie distale de la tête et du corps de l'épididyme $(13,21)$. Mais il faut souligner que chez les patients ayant subi avec succès une anastomose épididymo-déférentielle, le taux de grossesses est d'autant plus élevé que le site de l'anastomose est plus distal $(20,21)$.

\section{ANDROGENO-DÉPENDANCE DES FONCTIONS ÉPIDIDYMO-DÉFÉRENTIELLES}

L'intégrité morphologique et fonctionnelle du conduit épididymo-déférentiel est en premier lieu assurée par les androgènes. Ceci n'exclut pas pour autant la participation d'autres hormones véhiculées par le sang circulant (oestrogènes, aldostérone, prolactine, vitamines A7, D3). D'autre part, en complément du contrôle endocrinien, des facteurs paracrines non stéroïdes d'origine testiculaire paraissent impliqués dans le contrôle de la synthèse de protéines ou d'enzymes épididymaires. Cette action est particulièrement manifeste dans le cas de la régulation de l'activité de la $5 \alpha$ réductase.

L'épididyme reçoit les androgènes de la circulation périphérique et aussi du liquide en provenance du testicule. La testostérone et la DHT, dont les concentrations sont 5 à 50 fois plus élevées dans le liquide testiculaire que dans le sang, sont véhiculées vers l'épididyme sous forme libre grâce à l'ABP. La captation de la testostérone et sa conversion en $5 \alpha$ DHT est plus abondante dans la tête épididymaire où la concentration de l'ABP est maximale. Le complexe $\mathrm{ABP}$-testostérone est internalisé par endocytose dans les cellules épithéliales et la testostérone est convertie en DHT par la $5 \alpha$ réductase. La distribution de cette enzyme dans l'épithélium varie en fonction de l'âge et du niveau anatomique de l'épididyme. Comme dans tous les organes cibles, la DHT se lie à un récepteur caractérisé par une affinité élevée et une vitesse lente de dissociation. Le complexe hormone-récepteur interagit avec l'accepteur nucléaire induisant la transcription d'ARNms spécifiques.

En présence des androgènes, l'épididyme tire la plus grande partie de son énergie de l'oxydation des lipides. En absence des androgènes, le métabolisme énergétique dépend essentiellement des hydrates de carbone, du fait de la réduction de la synthèse des lipides. Le transport actif des ions ainsi que les systèmes responsables du transfert de la carnitine et de l'inositol à travers l'épithélium épididymaire sont androgénodépendants. La synthèse et la sécrétion de nombreuses glycoprotéines épididymaires et l'activité de plusieurs enzymes sont aussi régulées pour une grande part par les androgènes, sans qu'il soit possible toutefois de déterminer si cette action est directe ou médiée par des protéines nouvellement synthétisées.

La DHT est le seul androgène indispensable à l'acquisition du pouvoir fécondant des spermatozoïdes. Chez l'animal adulte castré et supplémenté en testostérone, l'administration conjointe d'un inhibiteur de la $5 \alpha$ réductase entraîne une diminution du nombre des spermatozoïdes mobiles, du pourcentage d'ovocytes fécondés et du nombre de blastocytes. L'action des androgènes sur les spermatozoïdes se poursuit au cours du stockage dans la queue de l'épididyme, du transport et du stockage dans le canal déférent.

En conclusion, le conduit épididymo-déférentiel constitue une entité anatomo-fonctionnel- le, régulée majoritairement par les androgènes, et indispensable à la maturation des spermatozoïdes. Il représente un site extragonadique de choix pour le contrôle de la fertilité masculine.

\section{REFERENCES}

1- Alnann RP : A critical review of methods for evaluation of spermatogenesis from seminal characteristics. J Androl, 1981, 2: 37-58

2 - Blaquier JA, Cameo MS, Dawidowski A, Gonzalez Echeverria F, Sanjurjo C : On the role of epididymal factors in sperm maturation in man. In Perspectives in Andrology, Serio M, ed, Serono Symposia Publications, New York, Raven Press, $1989,53: 37-44$.

3 - Beckman WC Jr, Fried PA, Stafford SJ, Mickey DD : Autoradiographic localization of tritiumlabeled in human vas deferens. J Androl, 1986, 7 : 97-202.

4 - Chinoy NJ : Structure and physiology of mammalian vas deferens in relation to fertility regulation. J Biosci, 1985, 7: 215-221.

5 - Cooper TG : The Epididymis, Speam maturation and Fertilization. Springer Verlag Heidelberg, 1986.

6- Cooper TG : In defense of a function for the human epididymis. Fertil Steril, 1990, $54: 965$ 975.

7 - Cooper TG : Secretory proteins from the epididymis and their clinical relevance. Andrologia, 1990, 22: 155-165.

8- Dacheux JL, Chevrier C, Lanson Y : Motility and surface transformations of human spermatozoa during epididymal transit. Proc Nat Acad Sci, 1987, 513: 560-563.

9- Dadoune JP. Modifications nucléaires des spermatides et des spermatozoïdes. Evaluation de la maturité nucléaire. Contr. Fertil Sex, 1991 , 19: 833-839

10 - Delpech S, Lecomte P, Lecomte C : Etude in vitro chez l'homme de la liaison des spermatozoïdes épididymaires à la zona pellucida, J Gynecol Obstet Biol Reprod, 1988,17: 339-342.

11 - Felden F, Leheup B, Fremont $S$ et al. :The plasma membrane of epididymal epithelial cells has specific receptor which binds to androgen binding protein and sex steroid-binding protein. J Steroid Biochem Molec Biol, 1992, 42: 279-285

12 - Junera HR, Alfonsi MF, Fain-Maurel MA, Dadoune JP: Characterization of regional proteins in tissues and fluids in the human epididymis. Reprod Nutr Develop, 1988, 28: 1 267- 1273.

13 - Mathieu C, Guérin JF, Cognat M, Lejeune H, Pinatel MC, Lornage J. :Motility and fertilizing capacity of epididymal human spermatozoa in normal and pathological cases. Fertil Steril, 1992, 57: $871-876$. 
14 - Murakami M, Sugita A, Hamasaki M. The vas deferens in man and monkey: spermiophagy in its ampulla. In : Atlas of human reproduction by scanning electron microscopy. 1982 E. Hafez, P Keneman eds, Lancaster, Boston, the Hague MTP Press limited, pp : 187-195

15 - Nagdas SK, Skudlarek M, Orgebin-Christ MC, Tulsiani DRP : Biochemical alterations in the proacrosin-acrosin system during epididymal maturation of the rat spermatozoa. J Androl, 1992, 13: 36-43.

16- Orgebin-Christ MC. Physiologie de l'épididyme et maturation du sperme: état actuel des connaisances. Contr. Fertil Sex, 1986; 14: 487-495.

17 - Robaire B, Hermo L : Efferents ducts, epididymis and vas deferens: structure, functions and their regulation. In : The Physiology of Reproduction, E. Knobil, J. Neil et al eds, Raven Press New York, 1988, 1 : 999-1080.

18 - Ross P, Kan FWK, Antaki P, Vigneault N, Chapdelaine A, Roberts KD : Protein synthesis and secretion in the human epididymis and immunoreactivity with sperm antibodies. Mol. Reprod. Dev. 1990, $26: 1223$.

19 - Rowley MJ, Teshima F, Heller CG : Duration of transit of spermatozoa through the human male ductulal system. Fertil. Steril. 1970, 21: 390-395.

20 - Schoysman RJ, Bedford JM : The role of the human epididymis in sperm maturation and sperm storage as reflected in the consequences of epididymovasostomy. Fertil Steril, 1986, 46: 293-299.

21 - Silber SJ : Apparent fertility of human spermatozoa from the caput epididymis. J Androl, 1989. 10: 263-269.

22 - Taragnat $\mathrm{C}$, Berger $\mathrm{M}$, Jean $\mathrm{Cl}$. Identification and androgen-dependence of proteins in the mouse vas deferens. Int J Androl, 1986, 9: 299-311.

23 - Yeung $\mathrm{CH}$, Cooper TG, Senge $\mathrm{T}$ : Histochemical localization and quantification of $\alpha$-glucosidase in the epididymis of men and laboratory animals. Biol Reprod, 1990, 42 : 69-676.
RESUME : Le conduit épididymo-déférentiel assure non seulement le transport mais l'acquisition du pouvoir fécondant des spermatozoïdes. Les fonctions du compartiment épithélial jouent un rôle déterminant dans la composition du liquide intraluminal dans lequel les spermatozoïdes subissent des modifications structurales, biochimiques et métaboliques. Durant le transit dans l'épididyme, les spermatozoïdes acquièrent leur mobilité et l'aptitude à se fixer sur la zone pellucide et à féconder l'ovocyte. Chez l'homme, la maturation des spermatozoïdes peut se produire dans la portion plus proximale du conduit épididymaire. La régulation des fonctiolls de l'épididyme et du canal déférent ainsi que la maturation des spermatozoïdes sont sous le contrôle des androgènes. Mots clés : Epididyme - Canal déférent - Physiologie - Maturation des spermatozoïdes. Andrologie, 1992, 2 : 44-47.

\section{BOURSES DE VOYAGE OFFERTES A DES JEUNES PAR L'ISA POUR SE RENDRE AU CONGRES MONDIAL D'ANDROLOGIE DE TOKYO}

\section{(2 au 6 Mai 1993)}

L'International Society of Andrology offre 20 bourses de voyage de 750 \$ US pour se rendre à Tokyo. Elles sont réservées à des scientifiques jeunes, avec une préférence pour ceux venant des pays en voie de développement, mais possibilité d'attribution à des européens. La condition pour l'obtention de la bourse est la soumission d'un mini-poster pour le congrès. Les boursiers seront sélectionnés d'après la qualité scientifique de leur résumé. La date limite pour l'envoi des résumés est le 31 Décembre 1992.

Le premier auteur du mini-poster doit préciser son souhait de participer à la compétition pour l'obtention d'une bourse dans une lettre jointe au mini-poster et au formulaire d'inscription. Les bénéficiaires seront informés avant le congrès. 